\title{
Opportunistic Networks and Cognitive Management Systems for Efficient Application Provisioning in the Future Internet: An Overview
}

\author{
Andreas Georgakopoulos, Kostas Tsagkaris, Vera \\ Stavroulaki, Panagiotis Demestichas \\ Department of Digital Systems \\ University of Piraeus \\ Piraeus, Greece \\ Email: \{andgeorg, ktsagk, veras, pdemest\}@unipi.gr
}

\section{Klaus Moessner}

Faculty of Engineering \& Physical Sciences

University of Surrey

Guildford, Surrey, UK

Email:k.moessner@surrey.ac.uk

\author{
Jens Gebert \\ Alcatel-Lucent Deutschland AG \\ Stuttgart, Germany \\ Email: Jens.Gebert@alcatel-lucent.com
}

\author{
Oriol Sallent \\ Universitat Politècnica de Catalunya \\ Barcelona, Spain \\ Email: sallent@tsc.upc.edu
}

\author{
Marcin Filo \\ EIT+ Research Center \\ Wroclaw, Poland \\ Email: marcin.filo@eitplus.pl
}

\begin{abstract}
In the Future Internet era, it will be required to provide coordinated extensions of the infrastructure as a potential solution to matters such as coverage and capacity extension, infrastructure supported opportunistic ad-hoc networking and traffic aggregation in the access and backhaul network. Opportunistic networks are a solution to that direction. Opportunistic Networks are temporary, localized, operator-governed network segments that are created under certain circumstances. To that respect, opportunistic networks are dynamically created, managed and terminated. Mechanisms for the efficient, dynamic creation, management and termination of opportunistic networks are needed. These can be implemented through cognitive management functionality. Furthermore, various stakeholders such as network operators, end-users and content/ service providers can be benefited from the new network paradigm which will lead to increased revenue for the operator, better QoS for the end-users and new opportunities for the content/ service providers. Consequently, this work is based on the OneFIT project's solution which comprises opportunistic networks and cognitive management systems for the efficient application provisioning in the Future Internet.
\end{abstract}

Keywords-opportunistic networks; cognitive management systems; control channels; future internet

\section{INTRODUCTION}

Opportunistic networks (ONs) introduce an era of wireless communications where nodes and terminals engage occasional mobility and routing patterns are dynamically configured. It is assumed that $\mathrm{ONs}$ are operator-governed, temporary, coordinated extensions of the infrastructure. They are dynamically created, through operator spectrum/ policies/ information/ knowledge, in places and at the time they are needed to deliver multimedia flows to mobile users, in a most efficient manner. They can comprise network elements of the infrastructure, and terminals/ devices potentially organized in an infrastructure-less manner.

To that respect, mechanisms for the efficient, dynamic creation, management and termination of ONs are needed. These can be implemented through cognitive management functionality.

The concept of coexistence of ONs with network infrastructure is not new as it has been already empirically analysed in [1]. For example, the possibility of extending ad-hoc networks with the support of infrastructure, in the so-called hybrid networks, has also been considered in [2] as a way of improving the connectivity in large-scale adhoc networks. Also in [3] the benefits of using a hybrid network architecture over pure ad hoc wireless networks with no infrastructure are theoretically analysed in terms of throughput capacity increase. In these cases, the studies are based on a theoretical framework but neither architectural aspects nor considerations on the peculiarities of infrastructure-less networks formation for service provision are addressed.

In this context, the OneFIT project (Opportunistic networks and Cognitive Management Systems for Efficient Application Provision in the Future InterneT) focuses on the elaboration of an ON-based unified solution which takes into account the special nature of 
ONs and proposes integrated ON business scenarios for the further promotion of ONs to real life.

The rest of the paper is structured as follows. In Section II, business scenarios are briefly analyzed in order to provide later on the envisaged technical solution and the challenges as Section III indicates. Afterwards, Section IV proposes a high-level architecture with the main building blocks, while Section $\mathrm{V}$ discusses the main business benefits and the expected outcome of each proposed scenario. Future steps which should be taken into consideration are available in the concluding Section VI.

\section{BUSINESS SCENARIOS}

For the further development and implementation of the ON concept, specific business scenarios have been considered as part of our approach. Specifically, main scenarios include the opportunistic coverage extension, the opportunistic capacity extension, the infrastructure supported opportunistic ad-hoc networking, the opportunistic traffic aggregation in the radio access network and the opportunistic resource aggregation in the backhaul network. The envisaged technical solution and the challenges of each scenario are addressed in the next section.

\section{A. Opportunistic coverage extension}

The rationale of the opportunistic coverage extension scenario lies on the fact that a device which acts as a traffic source like a laptop or a camera is out of the coverage of the infrastructure. As a result, a solution would comprise the creation of an $\mathrm{ON}$ in order to serve the out of infrastructure coverage source. The notion of opportunism primarily lies in i) the selection for participation in the $\mathrm{ON}$ of the appropriate subset of nodes, among candidate nodes that happen to be in the particular area, based on profile and policy information of the operator, and ii) the use of spectrum that will be designated by the operator, for the communication of the nodes of the opportunistic network.

\section{B. Opportunistic capacity extension}

In the opportunistic capacity extension scenario, it is assumed that a specific area which experiences traffic congestion issues can be off-loaded with the creation of an $\mathrm{ON}$ in order to re-route the traffic to non-congested Access Points (APs). This scenario enables devices to maintain the required level of QoS for a wireless communication link even though a congestion situation occurs. In particular, the following two types of congestion situations are considered: i) a system operating in a licensed/ unlicensed band is overloaded and cannot guarantee the provision of the required QoS anymore. In this case, the traffic can be re-distributed to neighboring uncongested cells (which can use different Radio Access Technologies -RATs) and ii) a system operating in an unlicensed band (such as Wi-Fi, etc.) or licensed band (such as femto Base Station (BS) in a randomly deployed, dense environment) is experiencing high levels of interference, since neighboring APs/BSs are accessing an identical part of the spectrum. Due to this problem, the link throughput is greatly decreased and a congestion situation occurs. As a result, the origin of the interference is identified and the concerned APs/BSs are reconfigured in order to avoid the congestion situation if this is possible.

\section{Infrastructure supported opportunistic ad-hoc networking}

The scenario, considers a completely infrastructureless, but still operator-governed $\mathrm{ON}$. The rationale for building the $\mathrm{ON}$ in this case is to exploit the fact that often the end-points of an application are "closely" located so that traffic exchange can be limited within its scope. That would result to a potential reduction of the traffic load that has to flow through the infrastructure. The target applications of the infrastructure supported opportunistic ad-hoc networking scenario are those involving intensive multimedia exchanges between end users located close to each other and applications such as network-enabled gaming etc.

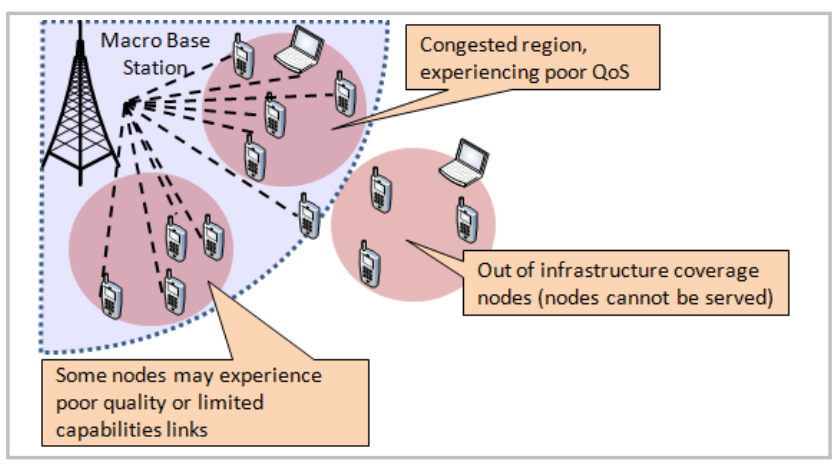

(a)

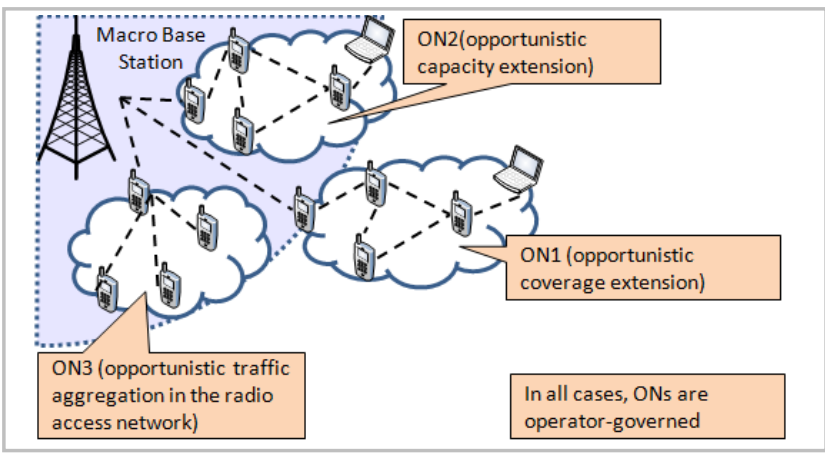

(b)

Figure 1. (a) Various problematic situations which may occur to a network, without the use of opportunistic networks, (b) Solutions to network issues by incorporating the indicative business scenarios of the opportunistic networks

\section{Opportunistic traffic aggregation in the radio access network}

The basic concept of this scenario lies on the fact that there is a certain concentration of users in a certain service area region that request a set of applications. Therefore, the infrastructure is required. On the other hand, the operator drives the users that are in the particular service area region into forming an $\mathrm{ON}$ with, at least, one network element of the infrastructure which can act as a gateway between the infrastructure and the $\mathrm{ON}$. The formed $\mathrm{ON}$ will comprise a BS providing macro-cell (or femtocell) coverage and a set of served devices, a subset of which is organized in an adhoc network mode. As a result an $\mathrm{ON}$ is created, in order to enable traffic aggregation from the $\mathrm{ON}$ to infrastructure 
through the gateway instead of having direct links from all nodes to the Macro BS. As a result, traffic exchange with the infrastructure via a limited number of users/ devices may yield improvement of the utilization of resources (assignment of fewer resources, better utilized, compared to the assignment of resources to all users).

\section{E. Opportunistic resource aggregation in the backhaul network}

The idea of this scenario is that an $\mathrm{ON}$ is created across multiple APs in order to primarily aggregate backhaul bandwidth and match the bandwidth of modern wireless access technologies towards the user with the adequate bandwidth on the backhaul/ core network $(\mathrm{CN})$ side. The same $\mathrm{ON}$ can be used to pull together processing or storage resources across multiple APs in order to pre-process user generated content and relieve pressure on the bandwidth resources needed for its transmission or the storage.

\section{ENVISAGED TECHNICAL SOLUTION AND CHALLENGES}

The proposed ON approach is based on four discrete phases. These phases include the ON suitability determination, the $\mathrm{ON}$ creation, the $\mathrm{ON}$ maintenance and the ON termination. Specifically, the ON suitability determination deals mainly with the node/ infrastructure discovery, the identification of candidate nodes and the identification of spectrum opportunities from the infrastructure side. The output of the suitability determination phase is expected to be the request for the creation of the $\mathrm{ON}$.

The creation phase follows, which is responsible for evaluating the data received from the suitability determination phase and then continues with the selection of the participant nodes, the selection of links/ spectrum /RATs and the routes. As a result, the $\mathrm{ON}$ is created. Main challenges of the creation phase are focused on the development of mechanisms that will lead to the interconnection of selected nodes, the optimization of routes, and usage of the selected spectrum followed by the signaling procedure establishing the ON. In some cases though, the creation phase could also come up with a decision for not finally establishing the ON.

Furthermore, in the post-creation stage, monitoring and possible reconfiguration procedures if QoS levels tend to drop significantly have to be dealt. For the monitoring and reconfiguration of the $\mathrm{ON}$, the responsibility lies in the maintenance phase. Main challenges of the maintenance phase will include the development of the proper mechanisms in order to handle the consistent monitoring of nodes, spectrum, policies, QoS and decide whether it is suitable to proceed to a reconfiguration of the $\mathrm{ON}$.

Finally, the termination phase can be distinguished into termination due to the cessation of application provisioning, termination due to inadequate gains from the $\mathrm{ON}$ or forced termination. The challenge of the first case is to successfully achieve the release of resources, while the latter two cases facilitate the handover to infrastructure as well, in order to maintain as flawless as possible, the application streams.

The technical challenges associated to the different phases outlined above will require the design of proper algorithmic solutions. The overall dynamic operation of the $\mathrm{ON}$ will be based on a comprehensive solution ensuring the integration and synergic operation among the different algorithms. For example, in the maintenance stage, the identification of spectrum opportunities functionality can dynamically monitor the status of diverse license and un-licensed bands in order to feed the spectrum selection decision-making process with updated information. In turn, the spectrum selection algorithm may decide e.g. a reconfiguration of the $\mathrm{ON}$ by executing a spectrum handover to a more suitable spectrum piece.

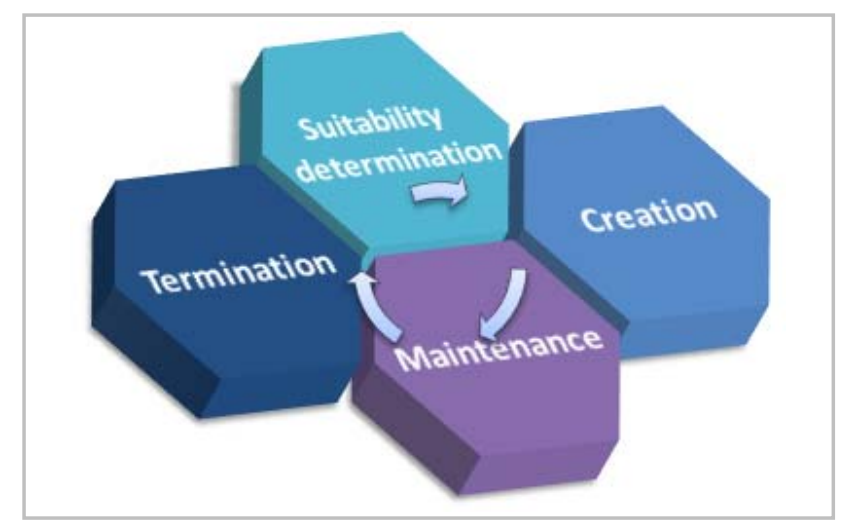

Figure 2. Opportunistic network phases.

\section{High-LEVEl FunCTIONAL ARCHITECTURE}

In order to address the identified ON approach and technical solution, there is a need for cognitive management systems combining self-management functionality and knowledge obtained through learning. In particular, two components/ sub-systems are required to be recognized as constituting entities of an ON management system. These are the Cognitive management System for the Coordination of the Infrastructure (CSCI) and the Cognitive Management system for the Opportunistic Network (CMON).

\section{A. Cognitive management System for the Coordination of the Infrastructure}

CSCI is the functional entity in charge of the context acquisition and processing, and the determination whether or not right conditions are in place for creating the $\mathrm{ON}$. The CSCI is deciding on (but not controlling of) the life cycle of the $\mathrm{ON}$ from the creation phase to a normal termination, as discussed earlier. The CSCI delegates actual creation, maintenance and termination of a given $\mathrm{ON}$ to the associated CMON functional entity. In case that the conditions (dictated by the policy engine) or the potential gains by the operation of the ON are satisfied, the CSCI will come up with an ON blue print design and pass it to the CMON for the execution. As CSCI is responsible for providing the interface between the overall system management system and the CMON it will also assist in the creation, maintenance and release phases. The CSCI should also be in charge of issuing and supervising a request for the forced $\mathrm{ON}$ termination. 


\section{B. Cognitive management System for the Opportunistic Network}

$\mathrm{CMON}$ on the other hand, is responsible for executing on the design obtained from the CSCI and then operationally supervising the created ON. This entity is in charge of the creation, maintenance and release (according to the policies maintained in the CSCI) of the ON. The contextual and performance parameters collected by the $\mathrm{CMON}$ during the life cycle of an $\mathrm{ON}$ are used for learning and improvement of its management functions/logic. Equally these data are passed onto the CSCI for improving the governance functions/logic hosted by the CSCI.

\section{Control Channels for the Cooperation of the Cognitive Management Systems}

The cooperation of the two cognitive management systems will require existence of well defined control messages and protocols/ channels for their exchange. That is the role of the Control Channels for the Cooperation of the Cognitive Management Systems $\left(\mathrm{C}^{4} \mathrm{MS}\right)$. The $\mathrm{C}^{4} \mathrm{MS}$ is responsible for managing the information exchange between functional entities involved in ON management. The $\mathrm{C}^{4} \mathrm{MS}$ can be seen as a combination and extension of the Cognitive Pilot Channel (CPC) [4] concept and the Cognitive Control Channel (CCC) concept (the CCC is described in [5] as a logical channel transporting information on top of a physical channel as e.g. provided by the Cognitive Control Radio -CCR). The CPC will be the basis for the coordination of the infrastructure with the ONs (communication between a CSCI and CMONs), while the CCR will be the basis for the exchange of cognition-related information between the peer nodes of the $\mathrm{ON}$ (communication between CMONs).

The management and control functionalities for ONs shall be an addition to existing functionalities in today's networks. Thus, the proposed Functional Architecture (FA) is an extension of an existing architecture, namely the "Functional Architecture for the Management and Control of Reconfigurable Radio Systems" as defined by ETSI in the TR102.682[6], [7]. The resulting FA is shown in Fig.3.

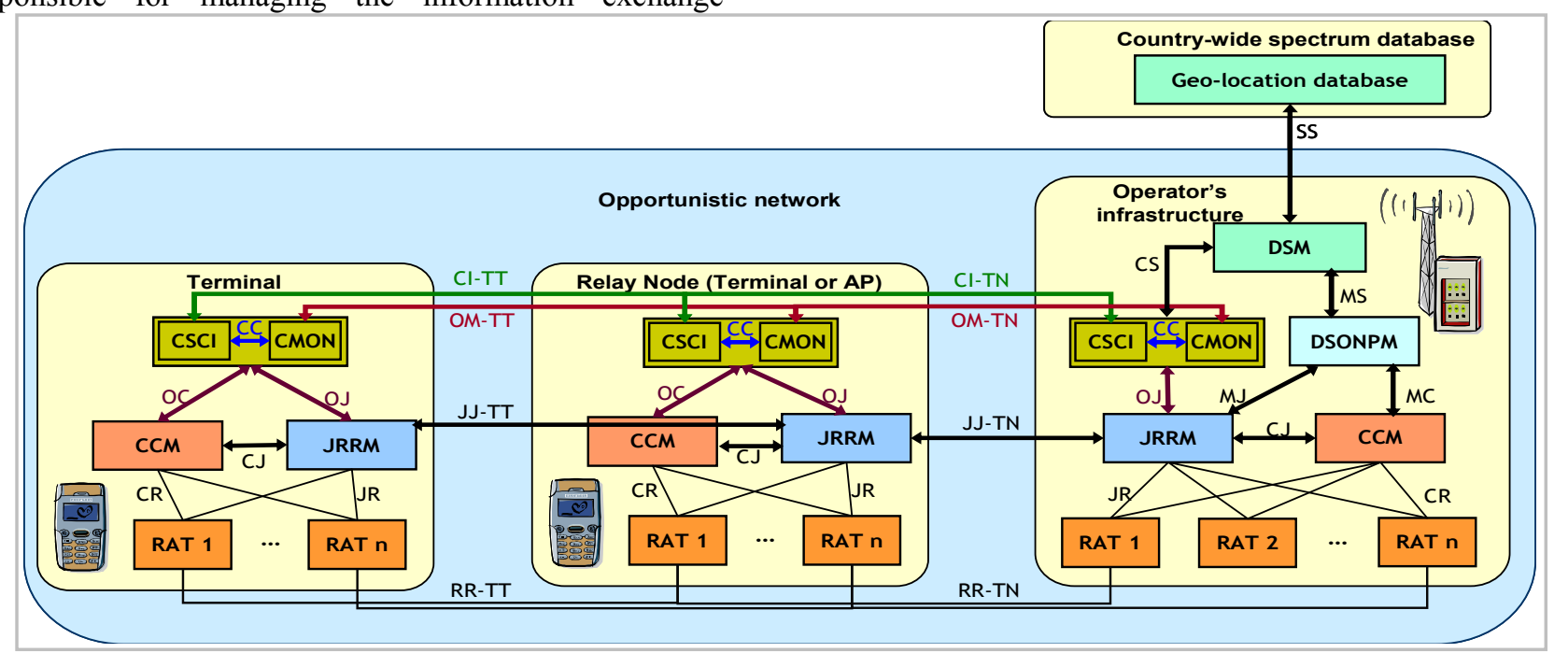

Figure 3. High-level Functional Architecture.

The main building blocks of the FA are:

- The CSCI and CMON.

- The Dynamic Spectrum Management (DSM) which provides mid- and long-term management (e.g. in the order of hours and days) of the spectrum for the different radio systems (extensive research activities have dealt with the identification of spectrum opportunities in [8], [9], [10], [11], [12], and management [13] ).

- The Dynamic, Self-Organising Planning and Management (DSONPM) which provides midand long-term decisions upon the configuration and reconfiguration of the network or parts of it. The DSONPM decides for example on the configuration of a base station and then instructs the CCM in order to execute the reconfiguration.

- The Joint Radio Resources Management (JRRM) which performs the joint management of the radio resources across different radio access technologies [14]. It selects the best radio access
(Access-Selection \& Handover Decisions) for a given user based on the session's requested QoS, radio conditions, network conditions like cell load, user preferences and network policies. The JRRM also provides Neighbourhood Information which can then be distributed via CCC or a CPC.

- The Configuration Control Module (CCM) which is responsible for executing the reconfiguration of a terminal or a base station, following the directives provided by the JRRM or the DSONPM.

These building blocks act on top of existing Radio Access Technologies (RATs) and act in whole or in part in both network and terminal sides, as shown in Fig. 3.

\section{EXPECTED OUTCOME AND BUSINESS BENEFITS}

Numerous benefits for different stakeholder categories can be derived from the ON approach, with respect to each scenario. Specifically, the coverage extension capability of an $\mathrm{ON}$ enables end users to gain access to the 
infrastructure in situations where it normally would not be possible, while the access provider may experience increased cashflow as more users are being supported, without at the same time being necessary to invest in new and rather costly infrastructure.

From the opportunistic capacity extension, access providers benefit from the fact that more users can be supported since new incoming users who otherwise would be blocked, can now be served, while end users experience improved QoS since congestion situations can be resolved. Additionally, infrastructure nodes should be able to save resources that could be used with new users, enhancing the service level of current ones, or just reducing their energy consumption. When envisioned solution is fully deployed, statistical gains in capacity/ coverage/ load management with a given dual-layer (macro \& femto) network are expected, allowing for better Return On Investment (ROI).

With respect to the infrastructure supported opportunistic ad-hoc networking, a potential reduction of the traffic load (user/ control planes) that has to go through the infrastructure is expected as an $\mathrm{ON}$ is created to serve closely located nodes which share common application interests. Other expected benefits from the infrastructure supported opportunistic ad-hoc networking approach are the reduction of the energy consumption of involved devices through the reduced required transmission power and the reduction of interference which can lead to a more efficient frequency reuse.

The exploitation of an $\mathrm{ON}$ solution with respect to the traffic aggregation in the radio access network includes lower transmission powers in the infrastructure (and therefore, reduced operational expenditures), similar volume of traffic served with less signaling traffic going through the infrastructure and higher utilization of resources (e.g., spectrum, therefore, higher capacity levels) without investing in the infrastructure. This leads to reduced capital expenditures with at least equivalent QoE/QoS levels.

Finally, main business benefits from the opportunistic resource aggregation in the backhaul network concept include that end users are experiencing better QoS in situations where backhaul would normally be congested, while access providers can make more efficient use of their network resources and generate increased revenue. When the resource aggregation in the backhaul network solution is fully deployed, it is expected that the overall capacity of the system to serve rich data applications should be increased. The concept will become increasingly attractive as the wireless system solutions become more heterogeneous and incorporate micro/ femto underlay based on Wi-Fi and femto cellular AP technologies.

\section{CONCLUSION AND FUTURE WORK}

This work presents a common ground to further study and elaborate the introduction of ONs to the implementation for efficient application provisioning in the Future Internet era. Additionally, five different scenarios, where ONs may pose a promising solution have been introduced. Derived from the scenarios, the envisaged technical solution and challenges have been identified along with the high-level description of cognitive management systems and the functional architecture.
Particularly, the functional architecture has been presented as an evolution of the previously developed ETSI RRS functional architecture for the Management and Control of Reconfigurable Radio Systems. Upcoming research will be focused on the described scenarios, including the development of the demonstration platforms for validation and proof-of-concept.

\section{ACKNOWLEDGMENT}

This work is performed in the framework of the European-Union funded project OneFIT (www.ictonefit.eu). The project is supported by the European Community's Seventh Framework Program (FP7). The views expressed in this document do not necessarily represent the views of the complete consortium. The Community is not liable for any use that may be made of the information contained herein.

\section{REFERENCES}

[1] P. Hui, A. Lindgren, J. Crowcroft, "Empirical evaluation of hybrid opportunistic networks", in Proc. of Communication Systems and Networks and Workshops, (COMSNETS 2009), Bangalore, India, January 2009

[2] O. Dousse, P. Thiran, M. Hasler, "Connectivity in ad-hoc and hybrid networks", in Proc. of the IEEE Computer and Communication Societies (Infocom 2002), New York, USA, June 2003

[3] U. Kozat, L. Tassiulas, "Throughput capacity of random ad hoc networks with infrastructure support", in Proc. of the 9th international conference on Mobile computing and networking, (Mobicom2003), 2003

[4] ETSI TR 102.683 “Cognitive Pilot Channel”

[5] $\mathrm{E}^{3}$ Deliverable D4.4 "Final solution description for autonomous CR functionalities", September 2009

[6] ETSI, TR 102.682 "Functional Architecture for the Management and Control of Reconfigurable Radio Systems"

[7] ICT-2007-216248 End-to-End efficiency ( $\left.E^{3}\right)$ Project, https://icte3.eu/

[8] D. Cabric, A. Tkachenko, R. W. Brodersen, "Spectrum sensing measurements of pilot, energy, and collaborative detection", in Proc. of Military Communications Conference, (MILCOM 2006), Washington DC, USA, October 2006.

[9] Z. Ye, J. Grosspietsch, G. Memik, "Spectrum sensing using cyclostationary spectrum density for cognitive radio", in Proc. of IEEE Workshop on Signal Processing Systems (SiPS 2007), Shanghai, China, October 2007

[10] M. Matinmikko, M. Mustonen, H. Sarvanko, M. Hoyhtya, A. Hekkala, A. Mammela, M. Katz, M. Kiviranta, "A motivating overview of cognitive radio, foundations, regulatory issues and key concepts", in Proc. of the First International Workshop on Cognitive Radio and Advanced Spectrum Management (CogART 2008), Aalborg, Denmark, February 2008

[11] A. Ghasemi, E.S. Sousa, "Spectrum sensing in cognitive radio networks: requirements, challenges and design trade-offs," IEEE Communications Magazine, Vol.46, No.4, pp.32-39, April 2008

[12] S. Mubaraq, R.W. Brodersen, S.T. Brink, R. Mahadevappa, "Detect and avoid: an ultra-wideband/WiMAX coexistence mechanism", IEEE Communications Magazine, Volume 45, Issue 6, June 2007

[13] J. Zhao, H.Zheng, G-h Yang, "Distributed coordination in dynamic spectrum allocation networks", in Proc. of Dynamic Spectrum Access Networks Conference, (DySPAN 2005), Baltimore, Maryland, USA, 2005

[14] H.-P. Shiang, M. van der Schaar, "Delay-sensitive resource management in multi-hop cognitive radio", IEEE Trans. on Vehicular Technology, Vol. 58, No. 2, February 2009 